\title{
Chronic prostatitis/chronic pelvic pain syndrome-related pain symptoms and their impact on sexual functioning
}

Benjamin Evans-Durán ${ }^{1}$; Dean A. Tripp ${ }^{2}$; Jeffrey Campbell³; R. Christopher Doiron ${ }^{4}$; J. Curtis Nickel ${ }^{4}$

${ }^{1}$ Department of Psychology, Queen's University, Kingston, ON, Canada; ${ }^{2}$ Department of Psychology, Anesthesia, Urology, Queen's University, Kingston, ON, Canada; ${ }^{3}$ Department of Surgery, Division of Urology, Western University, London, ON, Canada; ${ }^{4}$ Department of Urology, Queen's University, Kingston, ON, Canada

Acknowledgements: The authors would like to acknowledge Mr. Karl Monahan, founder of the Pelvic Pain Clinic U.K., who assisted in the distribution of study recruitment materials and offering a patient focused perspective.

Cite as: Evans-Durán B, Tripp DA, Campbell J, et al. Chronic prostatitis/chronic pelvic pain syndrome-related pain symptoms and their impact on sexual functioning. Can Urol Assoc J 2022 January 27; Epub ahead of print. http://dx.doi.org/10.5489/cuaj.7607

Published online January 27, 2022

Corresponding author: Mr. Benjamin Evans-Durán, Department of Psychology, Queen's University, Kingston, ON, Canada; ben.evansduran@queensu.ca

$* * *$

\section{Abstract}

Introduction: The present study sought to examine a new model to evaluate the mechanistic pathways between pain and sexual dysfunction in men with chronic prostatitis/chronic pelvic pain syndrome (CP/CPPS), incorporating cognitive and social factors.

Methods: Men with a self-reported diagnosis of CP/CPPS ( $\mathrm{n}=94,24-69$ years, $\mathrm{M}_{\mathrm{age}}=44.22$, standard deviation 11.25) were recruited through social media, support groups, and urology clinics and completed an online questionnaire of demographic, pain, cognitive, psychological, and sexual variables. Descriptive statistics, correlation analysis, and serial mediation analyses assessed variable associations.

Results: Almost half of participants reported mild to severe erectile dysfunction (47.4\%). Sexual dysfunction was associated with greater pain symptom severity and pain catastrophizing, as well as depressive symptoms ( $\mathrm{p}<0.01$ for all). While pain did not independently predict levels of sexual dysfunction, the addition of pain catastrophizing and depressive symptoms into the pathway explained the association between increased pain symptoms and decreased sexual functioning $(\mathrm{p}<0.01)$. 
Conclusions: Beyond generally poor sexual functioning in the current sample, it appears as if cognitive and emotional factors play a role in the association between pain symptoms and sexual functioning in these men with CP/CPPS. The findings of how pain catastrophizing and depression impact the association of pain severity and decreased sexual functioning is important for improving patient care.

\section{Introduction}

Chronic prostatitis/chronic pelvic pain syndrome (CP/CPPS) is associated with penile, testicular, ejaculatory, and/or perineal pain symptoms, ${ }^{1}$ and can impact patients' sexual activities and satisfaction. Indeed, sixty-two percent of patients with CP/CPPS report sexual dysfunction (i.e., ejaculatory difficulties, erectile dysfunction). ${ }^{2}$ Individuals with CP/CPPS with associated sexual dysfunction report a significantly worse quality of life (QoL) than those without. ${ }^{3}$ Additionally, individuals with $\mathrm{CP} / \mathrm{CPPS}$ and erectile dysfunction (ED), report increased depressive symptoms and pain catastrophizing, ${ }^{4}$ implicating cognitive and emotional factors in the relationship between $\mathrm{CP} / \mathrm{CPPS}$ symptoms and sexual functioning. To our knowledge, no study to date has tested a model including these parameters.

Cognitive emotional models of sexual dysfunction have been validated in populations with a clinical diagnosis of a sexual dysfunction, and those who experience subclinical sexual difficulties. Such models suggest that when individuals experience interference in their typical sexual functioning, they will display a heightened focus on the negative consequences of not performing typically, resulting in negative emotionality. ${ }^{5}$ The physiological pathway from pain in the penis and pelvis, and impaired erectile functioning is apparent and involves avoidance behaviours, but the psychological pathways underpinning this relationship remain uncertain. It is known that patients with CP/CPPS demonstrate elevations in depression, ${ }^{6}$ as well as pain catastrophizing, ${ }^{7}$ factors which have been found to predict sexual dysfunction within cognitiveemotional investigations of sexual dysfunction of individuals without CP/CPPS. ${ }^{8}$ It is not known whether the same cognitive-emotional pathways and mechanisms operative in those without a genitourinary and pelvic pain condition can be applied to urological patients complaining of these symptoms.

The objectives of this study were to assess a cognitive-emotional CP/CPPS model for the prediction of sexual dysfunction, using theoretically and clinically relevant variables. It was hypothesized that $\mathrm{CP} / \mathrm{CPPS}$ pain would have a direct positive relationship with sexual dysfunction and that this relationship would be mediated by pain catastrophizing and depressive symptoms. 


\section{Method}

\section{Participants}

Participants with a self-identified diagnosis of CP/CPPS were recruited (see "Procedure"). All participants had to be above the age of 18 years, and able to read and understand English. Participants completed an anonymous online survey through the Qualtrics platform (Qualtrics, Provo, UT).

\section{Measures}

Participants were asked to indicate their age, gender identity, country of residence, race/ethnicity, level of education, relationship, and employment status, and whether they have previously received a diagnosis of $\mathrm{CP} / \mathrm{CPPS}$.

The National Institutes of Health Chronic Prostatitis Symptom Index (NIH-CPSI), ${ }^{9}$ was used to assess CP/CPPS symptom severity. The commonly used 13-item measure includes three subscales: pain symptoms, urinary symptoms, and quality of life. The scoring of the items varies, with ranges from $0-1$ to $0-10$. The scores were summed together to obtain a total NIH-CPSI score from 0 to 43, as well as a score for the 8-item pain subscale which ranges from 0 to 21 .

The extent to which individuals engage in catastrophic thinking about their pain was measured using the four-item version of the Pain Catastrophizing Scale (PCS-4).${ }^{10}$ From the full length 13-item PCS, one item assessing magnification ("I become afraid the pain will get worse"), one item assessing helplessness ("It's terrible and I think it's never going to get any better"), and two items assessing rumination ("I anxiously want the pain to go away", "I keep thinking about how badly I want the pain to stop") were retained. Each of these dimensions have been identified as being central to pain catastrophizing appraisal and mechanisms.

The Patient Health Questionnaire-9 (PHQ-9) ${ }^{11}$ assessed depressive symptomology. The nine-item measure is commonly used to screen for symptoms of Major Depressive Disorder based on the DSM-V criteria, asking individuals to indicate how often they have been bothered by problems such as "little interest or pleasure in doing things," on a 0 (Not at all) to 3 (Nearly every day) Likert scale. Responses to the nine items were summed to obtain a total PHQ-9 score, with higher scores indicating worse depressive symptom severity. The measure was originally a subscale within the full 16 -item measure used for the same purpose. ${ }^{11}$

Sexual functioning was assessed using the International Index of Erectile Function (IIEF). ${ }^{12}$ The questionnaire contains 15 items (e.g., "How do you rate your confidence that you could get an erection?") to which participants must respond on a five-point Likert scale from 1 (Almost never or never) to 5 (Almost always or always). The items assess sexual functioning in men across several domains: erectile function, orgasmic function, sexual desire, intercourse satisfaction, and overall satisfaction. ${ }^{12}$ 


\section{Procedure}

This research was approved by Queen's University Health Sciences Research Ethics Board. The anonymous survey was administered via the Qualtrics platform (Qualtrics, Provo, UT). The study followed university mandated COVID-19 clinical research guidelines employing a multiformat sampling strategy. Participants were recruited via 4 avenues: 1) social media advertising, 2) The Pelvic Pain Clinic UK's newsletter, 3) referral from a urologist, and 4) snowball sampling. The social media advertising was conducted through Twitter, Facebook, Instagram, and Reddit. The participating urologists distributed physical advertisements with a QR code linking to the survey, directly in clinics and urology and pelvic pain specialists may have made personal referrals to the study's link through snowball sampling. Participants started the study by following the Qualtrics linked provided to them by the study advertisement and consented. All participants were asked whether or not they have received a diagnosis of $\mathrm{CP} / \mathrm{CPPS}$ and other demographic questions, and then completed all study measures. At any point during the study if a participant wished to stop, they could exit the study and terminate their participation in this anonymous study. No compensation was offered to participants.

\section{Data preparation}

The data was downloaded from the Qualtrics software (Qualtrics, Provo, UT). Before preparing the data, the dataset was reviewed for irregularities, such as missing or impossible values. For each given measure, only participants who completed $80 \%$ of the items would have their total scores calculated to be included in data analysis. ${ }^{13}$ Any missing data of a given measure was imputed and replaced with the means of all completed items on that measure. Upon completion, all total and subscale scores were calculated.

\section{Data analyses}

The demographics of the current sample, as well as mean scores on standardized measures were assessed. One-way analysis of variances (ANOVA), using Bonferroni correction, assessed mean differences in pain symptoms, pain catastrophizing, depressive symptoms, and sexual functioning across demographic variables. If mean differences were found, independent t-tests were performed to assess mean differences between groups.

Pearson's $r$ correlations were used to examine the relationship between sexual satisfaction and all independent variables to be used in a serial mediation analysis (i.e., age, pain symptoms, pain catastrophizing, depressive symptomology, sexual functioning). Based on the output of these correlations, variables that were not significantly associated with sexual functioning (i.e., outcome variable), were not included in the subsequent analyses.

To test the primary study hypothesis, a serial mediation assessed the relationship between pain symptoms and sexual functioning. This primary model was analyzed using model 6 from Hayes' PROCESS Macro for SPSS, ${ }^{14}$ with 2 mediators, 10,000 bootstrap samples, and 95\% confidence intervals. NIH-CPSI pain subscale scores will be input as the predictor variable, pain 
catastrophizing as the first mediator, depressive symptoms as the second mediator, and sexual functioning as the outcome variable.

\section{Results}

\section{Descriptive statistics}

Demographic characteristics of the sample are represented in Table 1. 42.9\% of those who opened the survey completed enough of the questionnaires to be included in the final analysis, resulting in a final sample of 97. All participants identified as male, and the average age of participants was $44.22(S D=11.25)$, ranging from 24-69 years of age. Majority of the sample self-identified as White (79.4\%), married (83.5\%), and having at least graduated from college/university (61.8\%). Most of the participants reported living in the United Kingdom, the United States, and Canada. 19.6\% of participants indicated that they were not from any of these countries $(n=19)$; Other than these 4 countries, participants indicated that they were living in Singapore, Italy, México, Philippines, Lithuania, Switzerland, Turkey, Guyana, Sweden, Spain, Iran, Germany, Egypt, Romania, South Africa, Iceland, and Hong Kong. 55.6\% of the sample were recruited via Facebook $(n=53), 2 \%$ from other social media platforms (i.e., Instagram, Reddit, Twitter; $n=2), 24.7 \%$ from a CP/CPPS support group $(n=24), 2 \%$ from a friend's referral $(n=2)$, and $13.4 \%$ received a referral from their urologist $(n=13)$.

There was one statistically significant difference between demographics and IIEF scores; race/ethnicity was the only demographic characteristic which displayed a significant relationship with sexual functioning $(p=.008)$. To further analyze this relationship, two categories for racial identity had to be created, due to some racial identities having two cases or less. Participants who indicated that they identified as 'White' were retained in this category $(n=77)$, and those who selected a racial identity other than white, were categorized as 'BIPOC' (Black, Indigenous, and People of Colour; $n=15$ ). After this recategorization, the groups showed significantly different means in IIEF scores $(p=.014)$, with white participants demonstrating worse sexual functioning $(M=51.10)$, compared to BIPOC participants $(M=38.07)$. There were no additional significant differences across education, relationship, and employment status, or where the participants had heard about the study from.

The 4-item PCS-4, 9-item PHQ-9, and 15-item IIEF demonstrated high internal consistency ( $\alpha=.82, .91$, and .93 respectively). The 13-item NIH-CPSI also demonstrated sufficient internal consistency $(\alpha=.78)$. The mean total score of the NIH-CPSI $(25.90, S D=$ 6.85 ) indicating moderate to severe $\mathrm{CP} / \mathrm{CPPS}$ severity, with $22.7 \%$ reporting severe, $61.9 \%$ moderate, and $4.1 \%$ reported mild symptoms. The mean score on the pain subscale was 12.31 $(S D=3.58)$. The mean PHQ-9 score $(11.16, S D=7.07)$ indicates minimal to moderately severe depressive symptoms overall; $16.5 \%$ of the sample reported minimal depressive symptom severity, $32 \%$ reported mild symptom severity, $18.6 \%$ reported moderate symptom severity, $19.6 \%$ reported moderate-to-severe symptom severity, and $13.4 \%$ reported severe depressive 
symptom severity. The mean score of the PCS-4 was $10.10(S D=3.92)$. The global score of the IIEF was $49.13(S D=18.66)$. To analyze the severity of $E D$, the erectile function domain of the IIEF was used and $47.4 \%$ of participants met criteria; $16.5 \%$ had mild ED, $12.4 \%$ had mild-tomoderate ED, $10.3 \%$ had moderate ED, and $8.2 \%$ had severe ED.

\section{Correlations among variables}

As shown in Table 2, Pearson's $r$ correlations were used to analyze the extent to which the current study's variables were correlated. NIH-CPSI pain symptom, pain catastrophizing, depressive symptoms, and sexual functioning scores were all correlated. The largest correlation emerged between PCS-4 and PHQ-9 scores. IIEF and PHQ-9 scores were also correlated, indicating that sexual functioning is more highly correlated with depressive symptoms than the other predictor variables. Sexual dysfunction was negatively correlated with pain symptom, pain catastrophizing, and depressive symptom scores, indicating that greater scores on all these variables are related to worse sexual functioning.

\section{Serial mediation}

The serial mediation analysis determined the relationship between CP/CPPS pain symptoms and sexual dysfunction, through the indirect effects of pain catastrophizing, and depressive symptomatology. As expected, the total effect of NIH-CPSI pain symptomatology on sexual functioning was significant, $b=-1.72, S E=0.50,95 \% \mathrm{CI}[-2.721,-0.717]$, suggesting that the combined weights of pain symptoms, pain catastrophizing, and depressive symptoms were significantly associated with sexual functioning in patients with CP/CPPS. The direct effect from $\mathrm{CP} / \mathrm{CPPS}$ pain symptomatology to sexual functioning was much smaller and did not reach statistical significance (pain symptoms $\rightarrow$ sexual functioning), $b=-0.64, S E=0.54,95 \%$ CI [$1.716,0.440]$. The individual unstandardized path coefficients for the predictor variables and sexual functioning are illustrated in Figure 1.

While pain symptoms in isolation were not related to sexual functioning scores, when the mediating influences of pain catastrophizing and depressive symptoms were accounted for (pain symptoms $\rightarrow$ pain catastrophizing $\rightarrow$ depressive symptoms $\rightarrow$ sexual functioning), pain symptoms were associated with sexual functioning, $b=-0.48, S E=0.20,95 \%$ CI [-0.939, $0.163]$. Increased pain symptom scores led to increased pain catastrophizing levels, leading to increased depressive symptoms, and ultimately resulting in decreased sexual functioning scores. Depression on its own also mediated the relationship between pain symptom and sexual dysfunction scores (pain symptoms $\rightarrow$ depressive symptoms $\rightarrow$ sexual functioning), $b=-0.66, S E=0.25,95 \%$ CI $[-1.20,-0.25]$. Pain catastrophizing was not found to mediate the relationship independent of depressive symptoms scores, $b=0.06, S E=0.26,95 \%$ CI [-0.515, $0.550]$. 


\section{Discussion}

The relationship between pain and sexual functioning is best explained through pain catastrophizing and depressive symptoms. The serial mediation analysis revealed that pain symptoms in isolation do not effectively predict sexual functioning scores in this sample yet did when influences of pain catastrophizing and depressive symptoms were entered into the model as mediators. Depressive symptoms were also found to be an effective mediator without the influences of pain catastrophizing, but pain catastrophizing did not emerge as a unique mediator. These results provide evidence that pain is not the primary driver of sexual dysfunction in $\mathrm{CP} / \mathrm{CPPS}$ but supports a much more complex role of cognitive-emotional processing in impaired sexual functioning.

Previous studies have found that patients with CP/CPPS and ED demonstrate higher levels of depressive symptoms, pain catastrophizing, and symptom severity compared to those without ED, ${ }^{4}$ prompting the use of such variables in the present study. The association of poor sexual functioning with cognitive factors and emotional states is consistent with prior research in men with genital pain. ${ }^{15,16}$

Prior CP/CPPS literature has failed to examine the relationship that cognitive-emotional variables have with each other and how these factors predispose patients to experiencing a decrease in sexual functioning. The mediation analysis results suggest that when individuals experience pain, predominantly in the pelvic region, they begin to catastrophize the extent of their pain and ruminate on this pain's impact on their daily life and typical functioning, such as sexual functioning. Then, negative emotions are experienced because of this attentional focus on negative consequences from failure to function typically, and the individuals' functioning further decreases. ${ }^{17}$ The specific aspect of functioning examined in the present study was sexual functioning, such as erectile and orgasmic function. The novel mediation model tested in this study offers a new insight into the directionality of how pain symptoms and cognitive-emotional processes lead to poor sexual functioning in the sample.

The present primary line of treatment for ED in CP/CPPS includes the administration of phosphodiesterase type 5 (PDE-5) inhibitors, ${ }^{18}$ which may improve the physiological components of ED yet neglect the omnipresent mental components. The present study's evidence for the role that psychological factors have in perpetuating poor sexual functioning in patients with $\mathrm{CP} / \mathrm{CPPS}$ offers evidence for the incorporation of psychotherapy into current treatment standards, such as cognitive behavioural therapy (CBT). CBT in conjunction with pharmaceutical agents has been shown to lead to extended improvements upon sexual parameters in men with ED, compared to the use of pharmaceutical agents alone. ${ }^{18}$ In a feasibility trial, Nickel et al. ${ }^{19}$ found that a cognitive behavioural symptom management program for CP/CPPS significantly reduced patients' levels of pain catastrophizing, disability, and pain, but not depressive symptoms. This program challenged patients' illness focused coping and catastrophic cognitions, potentially explaining the improvements in catastrophizing but not depressive 
symptomatology, although the two have been found to be highly related. ${ }^{5}$ Thus, future multimodal therapies for CP/CPPS should incorporate considerations of depressive symptoms in the condition and include evidence-based methods of reducing depression through CBT identified in healthy individuals. $^{20}$

There are study limitations to mention. Due to the largely homogenous racial distribution of participants in the present study, the results may not be generalizable to BIPOC (Black, Indigenous, and People of Colour) individuals may only be generalizable to White individuals with CP/CPPS. Additionally, the survey did not include assessment of sexual orientation of participants, negating the opportunity to assess differences across sexual identities. Pre-COVID surveys that were conducted online have been routinely critically appraised. But, due to restrictions in patient engagement and freedom of movement, online surveys have become a valued methodology. The current survey was administered online due to the difficulties posed to in-person recruitment following health and safety measures aimed at reducing the spread of COVID-19. While the participants' scores on the measures included in this study closely mirror those obtained from patients at tertiary care clinics, ${ }^{4}$ they were slightly elevated. This may be best explained by literature on self-report survey modes, which proposes that individuals may be more likely to disclose stigmatized behaviours (which could include the inability to obtain/maintain an erection, or feelings of sadness in men particularly) via computerized surveys, opposed to in-person survey administration. ${ }^{21}$ As usual, replication and extension of the current study is recommended.

The present findings suggested that men with $\mathrm{CP} / \mathrm{CPPS}$ are at risk of experiencing poor sexual functioning due to pain in the genitals, pain catastrophizing and depressive symptoms. The implication of cognitive and emotional factors in the etiology and maintenance of poor sexual functioning adds further support to the cognitive-emotional model of sexual dysfunction, begging future research to adapt comprehensive frameworks when studying sexuality. The evidence additionally adds support to the growing movement to integrate psychotherapy into the multifaceted management of CP/CPPS. 


\section{References}

1. Krieger JN, Egan KJ, Ross SO, et al. Chronic pelvic pains represent the most prominent urogenital symptoms of "chronic prostatitis." Urology 1996; 48: 715-22.

https://doi.org/10.1016/s0090-4295(96)00421-9

2. Lee SW, Liong ML, Yuen KH, et al. Adverse impact of sexual dysfunction in chronic prostatitis/chronic pelvic pain syndrome. Urology 2008; 71: 79-84. https://doi.org/10.1016/j.urology.2007.08.043

3. Li HJ, \& Kang DY. Prevalence of sexual dysfunction in men with chronic prostatitis/chronic pelvic pain syndrome: a meta-analysis. World Journal of Urology 2015; 34: 1009-17. https://doi.org/10.1007/s00345-015-1720-3

4. Zhang Y, Zheng T, Tu X, et al. Erectile dysfunction in chronic prostatitis/chronic pelvic pain syndrome: Outcomes from a multi-center study and risk factor analysis in a single center. PLoS One 2016; 11. https://doi.org/10.1371/journal.pone.0153054

5. Barlow DH. Causes of sexual dysfunction: The role of anxiety and cognitive interference. Journal of Consulting and Clinical Psychology 1986; 54: 140-48. https://doi.org/10.1037/0022-006x.54.2.140

6. Kwon JK, \& Chang IH. Pain, catastrophizing, and depression in chronic prostatitis/chronic pelvic pain syndrome. International Neurology Journal 2013; 17: 48. https://doi.org/10.5213/inj.2013.17.2.48

7. Krsmanovic A, Tripp DA, Nickel JC, et al. Psychosocial mechanisms of the pain and quality of life relationship for chronic prostatitis/chronic pelvic pain syndrome (CP/CPPS). Canadian Urological Association Journal 2014; 8: 403. https://doi.org/10.5489/cuaj.2179

8. Peixoto MM, \& Nobre PJ. Cognitive-emotional predictors of sexual functioning in lesbians, gays, and heterosexuals. Archives of Sexual Behavior 2020; 49: 1823-38. https://doi.org/10.1007/s10508-020-01732-9

9. Litwin MS, Mcnaughton-Collins M, Fowler FJ, et al. The NIH Chronic Prostatitis Symptom Index (NIH-CPSI). The Journal of Urology 1999; 162: 369-75. https://doi.org/10.1097/00005392-199904010-00125

10. Sullivan MJL, Bishop SR, \& Pivik J. The Pain Catastrophizing Scale: Development and validation. Psychological Assessment 1995; 7: 524-32. https://doi.org/10.1037/10403590.7.4.524

11. Kroenke K, Spitzer RL, Williams JBW. The PHQ-9. Journal of General Internal Medicine 2001; 16: 606-13. https://doi.org/10.1046/j.1525-1497.2001.016009606.x

12. Rosen RC, Riley A, Wagner G, et al. The International Index of Erectile Function (IIEF): A multidimensional scale for assessment of erectile dysfunction. Urology 1997; 49: 82230. https://doi.org/10.1016/s0090-4295(97)00238-0

13. Tabachnick BG, Fidell LS, Ullman JB. Using multivariate statistics 2019. Pearson.

14. Hayes AF. Introduction to mediation, moderation, and conditional process analysis a regression-based approach (2nd ed.) 2018. The Guilford Press.

15. Aubin S, Berger RE, Heiman JR, et al. The association between sexual function, pain, and psychological adaptation of men diagnosed with chronic pelvic pain syndrome Type 
III. The Journal of Sexual Medicine 2008; 5: 657-67. https://doi.org/10.1111/j.17436109.2007.00736.x

16. Pereira R, Margarida Oliveira C, Nobre P. Sexual functioning and cognitions during sexual activity in men with genital pain: A comparative study. Journal of Sex \& Marital Therapy 2015; 42: 602-15. https://doi.org/10.1080/0092623x.2015.1113582

17. Nobre PJ. Psychological determinants of erectile dysfunction: Testing a cognitiveemotional model. The Journal of Sexual Medicine 2010; 7: 1429-37. https://doi.org/10.1111/j.1743-6109.2009.01656.x

18. Khan S, Amjad A, Rowland D. Potential for long-term benefit of cognitive behavioral therapy as an adjunct treatment for men with erectile dysfunction. The Journal of Sexual Medicine 2019; 16: 300-06. https://doi.org/10.1016/j.jsxm.2018.12.014

19. Nickel JC, Mullins C, Tripp, DA. Development of an evidence-based cognitive behavioral treatment program for men with chronic prostatitis/chronic pelvic pain syndrome. World Journal of Urology 2008; 26: 167-72. https://doi.org/10.1007/s00345008-0235-6

20. Fennell MJ. Cognitive behaviour therapy for depressive disorders. New Oxford Textbook of Psychiatry 2012; 1304-13. https://doi.org/10.1093/med/9780199696758.003.0167

21. Gnambs T, Kaspar K. Disclosure of sensitive behaviors across self-administered survey modes: a meta-analysis. Behavior Research Methods 2014; 47: 1237-59. https://doi.org/10.3758/s13428-014-0533-4

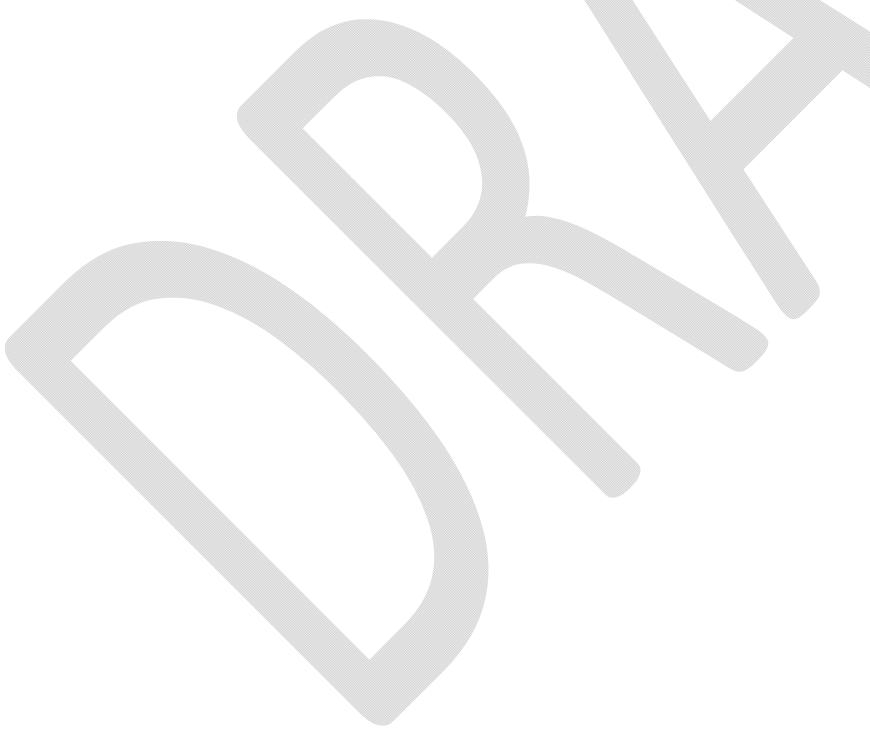




\section{Figures and Tables}

Fig. 1. Serial mediation model with chronic prostatitis/chronic pelvic pain syndrome (CP/CPPS) pain symptoms as the predictor variable and sexual function as the outcome variable.

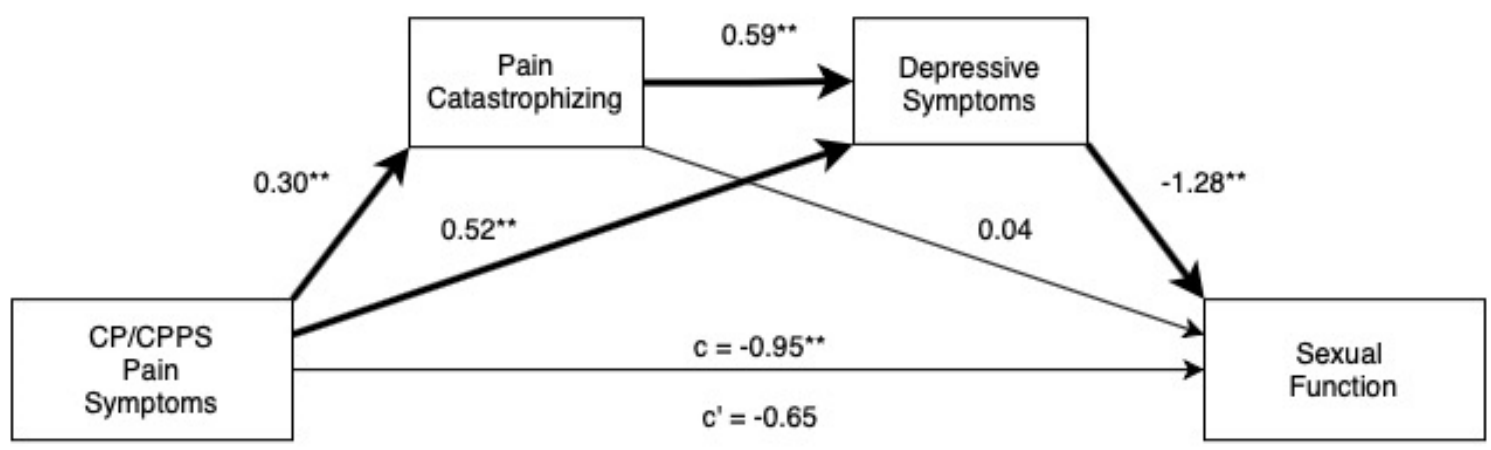

Note. Significant pathways are bolded. Significant coefficients are denoted by ${ }^{*}=p<.05,{ }^{* *}=p<.001$.

\section{Table 1. Demographic information of participants}

\begin{tabular}{|l|l|l|}
\hline Baseline characteristics & $\mathbf{n}$ & $\begin{array}{l}\text { Full sample } \\
\text { (\%) }\end{array}$ \\
\hline Race/Ethnicity & & \\
\hline Black & 2 & 2.1 \\
\hline Asian & 8 & 8.2 \\
\hline Hispanic/Latinx & 3 & 3.1 \\
\hline Indigenous & 1 & 1.1 \\
\hline White & 77 & 79.4 \\
\hline Other & 1 & 1.1 \\
\hline Country of residence & & \\
\hline Canada & 17 & 17.5 \\
\hline United States & 29 & 29.9 \\
\hline United Kingdom & 31 & 32 \\
\hline Australia & 1 & 1.1 \\
\hline Other & 19 & 19.6 \\
\hline Education status & & \\
\hline Some high school or GED & 12 & 12.4 \\
\hline Some college/university & 19 & 19.6 \\
\hline
\end{tabular}




\begin{tabular}{|l|l|l|}
\hline Graduate from college/university & 30 & 30.9 \\
\hline Some post-graduate & 5 & 5.2 \\
\hline Graduated from post-grad & 30 & 30.9 \\
\hline Employment status & & \\
\hline Employed & 83 & 85.6 \\
\hline Unemployed & 4 & 4.1 \\
\hline Retired & 8 & 8.2 \\
\hline Student & 2 & 2.1 \\
\hline Relationship status & & \\
\hline Single & 11 & 11.3 \\
\hline Married & 81 & 83.5 \\
\hline Divorced & 3 & 3.1 \\
\hline
\end{tabular}

GED: general educational development.

\begin{tabular}{|l|l|l|l|l|l|}
\hline \multicolumn{6}{|l|}{ Table 2. Correlation matrix for predictor variables and sexual functioning } \\
\hline $\begin{array}{l}\text { Relevant } \\
\text { variable }\end{array}$ & Age & Pain & $\begin{array}{l}\text { Depressive } \\
\text { symptoms }\end{array}$ & $\begin{array}{l}\text { Pain } \\
\text { catastrophizing }\end{array}$ & $\begin{array}{l}\text { Sex } \\
\text { function }\end{array}$ \\
\hline Age & - & & & & \\
\hline Pain & -0.12 & - & & & \\
\hline $\begin{array}{l}\text { Depressive } \\
\text { symptoms }\end{array}$ & -0.14 & $0.48^{*}$ & - & & \\
\hline $\begin{array}{l}\text { Pain } \\
\text { catastrophizing }\end{array}$ & -0.20 & $0.43^{*}$ & $0.60^{*}$ & - & \\
\hline Sex function & -0.07 & $-0.33^{*}$ & $-0.50^{*}$ & $-0.29^{*}$ & - \\
\hline
\end{tabular}

Pain: NIH-CPSI pain symptoms. ${ }^{*} p<0.01$ (two-tailed). 